\title{
FORMACIÓN DE DIRIGENTES GREMIALES MÉDICOS DE LA CIUDAD DE BUENOS AIRES EN CONDICIONES Y MEDIO AMBIENTE DE TRABAJO
}

\author{
TRAINING ON WORK AND ENVIRONMENTAL CONDITIONS FOR DOCTORS \\ UNION LEADERS OF BUENOS AIRES CITY
}

\author{
HÉCTOR ALBERTO NIETO $^{(1,3)}$; EZEQUIEL CONSIGLIO(2); MARIA JOSÉ ITATI IÑIGUEZ ${ }^{(1)}$; FABIANA \\ BALLADORE $^{(1)}$; MARÍA MARTA SAPOZNIK ${ }^{(3)}$, ILSE IRIATTI ${ }^{(3)}$, ALCIRA FIORINI $^{(4)}$; JORGE GILARDI ${ }^{(4)}$
}

(1) Cátedra Libre Salud y Seguridad en el Trabajo; Facultad de Medicina -

Universidad de Buenos Aires. Argentina.

(2) Departamento de Salud Publica; Facultad de Medicina - Universidad de Buenos Aires. Argentina.

(3) Servicio de Medicina del Trabajo; Hospital Parmenio Piñero - GCBA - de Buenos Aires. Argentina.

(4) Asociación de Médicos Municipales - Ciudad de Buenos Aires. Argentina.

\section{RESUMEN}

Objetivo: presentar la experiencia que la Asociación de Médicos Municipales (A.M.M) de la Ciudad de Buenos Aires desarrolló en materia de formación de delegados gremiales en prevención.

Materiales y métodos: La AMM convocó a un grupo técnico especializado que diseñó, desarrolló y evaluó el primer curso de formación de delegados en prevención de riesgos laborales en CyMAT para médicos. Se presenta la programación general de dicho curso, sus contenidos y actividades, y los resultados de su evaluación.

Resultados: Se presentan los resultados de las actividades de observación de los coordinadores del curso, los de una encuesta de evaluación a cargo de los alumnos y los emergentes cualitativos generales.

Se han identificado: la toma de conciencia por parte de los delegados médicos sobre los riesgos a los que se encuentran expuestos en su ámbito laboral, haber establecido vínculos y adquirido conocimiento de otras realidades, y el reconocimiento de la responsabilidad del empleador. Asimismo, surgieron propuestas de acción colectiva que se comenzaron a desarrollar como parte de las acciones gremiales.

Conclusión: Esta experiencia verifica la posibilidad de generar conocimiento traducido en acciones gremiales en el Sector Salud mediante el compromiso de los principales sujetos: los trabajadores médicos.

Palabras clave: Sector Salud, Condiciones y medioambiente de trabajo (CyMAT), Delegados de prevencion.

\section{ABSTRACT}

Objectives: to present the experience of the Asociación de Médicos Municipales (AMM) from the city of Buenos Aires developed for teaching and training of union delegates in prevention.

Materials and methods: The AMM convened a group of specialists for this goal, who have developed and evaluated this first training course for delegates at preventing occupational hazards in work-related environment and conditions of job for physicians. We present a general planning of the course and its schedule, its contents and activities, and the results of its assessment.

Results: The results of the monitoring activities by the coordinators of course, the scores of the survey answered by the delegates and emerging qualitative issues.

It have been identified some features: the awareness within delegates doctors about the risks to which they are exposed in their workplace, the links between themselves about their different realities and the acquired knowledge, and the recognition of the responsibility of the employer over the health of their workers. It also emerged that proposals for collective action began to develop as part of union action.

Conclusion: This experience demonstrates the ability to optimize the in health knowledge and use it in union action within the workers of the Health Sector.

Key words: Healthcare Sector, Environment of workplace and conditions of job, Prevention delegates. 


\section{INTRODUCCIÓN}

Bernardino Ramazzini de Módena - para muchos, padre de la Medicina del Trabajo - publica en 1700 su tratado "De morbis artificum diatriba"i. Su idea de escribir un tratado sobre las enfermedades de los trabajadores surge cuando observa lo penoso de la labor de un hombre dedicado al vaciamiento y limpieza de las cloacas: de esta actividad que el trabajador se ve obligado a realizar derivan molestias, enfermedades graves y muertes.

Basado en el estudio de cincuenta y tres oficios distintos define al trabajo como determinante del proceso salud-enfermedad-atención. Afirma en el prólogo de dicha obra "...Deberé confesar que ocasionan no poco daño a los obreros ciertos oficios que desempeñan: Donde esperaban obtener recursos para el propio mantenimiento y sostén familiar, hallan a menudo gravisimas enfermedades y maldicen el arte al que se habian dedicado mientras se alejan del mundo de los vivos...". ¿Qué hubiera observado, analizado y propuesto ante el trabajo médico?

En los siglos subsiguientes los cambios ocurridos en los sistemas de producción económica determinaron modificaciones en las exposiciones a factores de riesgo para el desarrollo de enfermedades. Durante la Revolución Industrial, se incorporaron materias primas, combustibles y maquinarias, que constituyeron nuevas fuentes de exposición para accidentes y enfermedades ligados al trabajo. Estos nuevos métodos de producción aumentaron exponencialmente la capacidad de generar bienes, al tiempo que muchos trabajadores - hombres, y también mujeres y niños - perdían su salud y su vida. Esta nueva evidencia, sobre el trabajo como determinante del proceso de salud-enfermedad-atención, requirió aún más tiempo para su consolidación como temática de Salud Pública. En este escenario se produjo la movilización de los trabajadores y su organización colectiva dando como resultado importantes transformaciones en materia de relaciones laborales, que se reflejaron en mejoras de las condiciones de trabajo. La incorporación de normas tendientes a aliviar la carga y los efectos del trabajo sobre la vida de los trabajadores y la preocupación por su salud, alcanzaron un lugar destacado.

Desde entonces, las normas internacionales en materia de protección de los derechos de los trabajadores adquirieron una mayor relevancia. En esta línea, se inscribe una de las últimas propuestas que la Organización Internacional del Trabajo (OIT) acuñó a fines de la década del `90: la noción de "Tra- bajo Decente" ii que revaloriza los derechos fundamentales de los trabajadores.

Los profesionales del Sector Salud se encuentran expuestos a una importante cantidad de agentes de riesgos cuyos efectos más frecuentes son la presencia del Síndrome de Desgaste Profesional (Burnout), el contagio de enfermedades infecciosas como tuberculosis y hepatitis virales; el padecimiento de diferentes formas de violencia; accidentes punzocortantes, intoxicaciones y cáncer entre otros.

La responsabilidad del empleador por la protección de la salud de los trabajadores se encuentra taxativamente enunciada en las leyes. Sin embargo, el nivel de cobertura especifica en Salud y Seguridad en el Trabajo entre los trabajadores del Sector Salud sigue siendo casi inexistente: es todavía excepcional la presencia de Servicios de Salud y Seguridad en los hospitales de la Ciudad de Buenos Aires.

La acción gremial organizada con base técnica y conocimiento científico, constituye un dispositivo idóneo para alcanzar el objetivo de Trabajo Decente. La participación activa de los trabajadores médicos en el mejoramiento de sus Condiciones y Medio Ambiente de Trabajo (CyMAT) impuestas, aparece como la forma más eficaz. A los fines de esta presentación entenderemos por CyMAT al conjunto de propiedades que caracterizan la situación de trabajo, influyen en la prestación del mismo y pueden afectar la salud de los trabajadores ${ }^{\text {iii }}$.

El presente trabajo tiene por objeto presentar a la comunidad médica internacional la experiencia que la Asociación de Médicos Municipales (A.M.M) de la Ciudad de Buenos Aires desarrolla en materia de formación de dirigentes gremiales en la prevención de riesgos laborales derivados de las CyMAT.

\section{MATERIALES Y MÉTODOS}

\section{Antecedentes históricos y programación}

La Asociación de Médicos Municipales agrupa a la casi totalidad de los médicos contratados por la administración de la Ciudad Autónoma de Buenos Aires. Sus actividades como entidad representativa del sector datan formalmente de 1936. Hacia mediados del año 2006 en respuesta a la demanda sostenida de los afiliados, sus autoridades decidieron realizar una Jornada de Reflexión y Debate acerca de la Participación de los trabajadores en la mejora de las Condiciones y Medio Ambiente de Trabajo ${ }^{\text {iv }}$ como mecanismo para la Prevención de Riesgos del 
Trabajo, basándose en el Convenio $155^{\vee}$ y la Recomendación $164^{\mathrm{vi}}$ de la Organización Internacional del Trabajo. Como parte de las conclusiones de aquel debate, surgió el mandato para que la Asociación de Médicos Municipales forme delegados de prevención en CyMAT. Por intermedio de su Secretaría de Asuntos Académicos convocó a un grupo técnico especializado en Salud y Seguridad en el Trabajo. El mismo estuvo integrado por cuatro médicos especialistas en Medicina del Trabajo, un médico Epidemiólogo, una Lic. en Psicología y una Lic. en Sociología, quienes tienen como antecedentes haber desarrollado el único grupo de Medicina del Trabajo en el sector público municipal desde 1994.

Los delegados de prevención en CyMAT - según las recomendaciones internacionales citadas - deben ser representantes de los trabajadores con funciones específicas en materia de prevención de riesgos en el trabajo. Pese a que esta figura aún no ha alcanzado un status legal en la Argentina, los destinatarios del curso fueron médicos que a la fecha de su inicio se encontraban desempeñando actividad gremial en las filiales de la AMM. Su designación fue determinada finalmente con el concurso de sus compañeros gremiales.

La Secretaria de Asuntos Académicos recibió 65 solicitudes de inscripción, representando a 28 filiales correspondientes a hospitales y otros centros de salud de segundo y tercer nivel de atención dependientes del Gobierno de la Ciudad Autónoma de Buenos Aires. Todas fueron aceptadas.

\section{Contenidos, objetivos, métodos}

El propósito del curso fue planteado en términos de “...Formar delegados de prevención en CyMAT con conocimientos, habilidades y destrezas que contribuyan, desde la acción sindical, a lograr la vigilancia -control y erradicación de los factores de riesgos presentes en el trabajo-; así como también facilitar el acceso de los trabajadores a los subsistemas de la seguridad social...".

Los objetivos específicos acordados fueron: (a) reconocer la interacción salud - trabajo; (b) identificar, reconocer y mapear los riesgos a los que se encuentran expuestos los trabajadores de la salud; (c) detectar, investigar y proponer soluciones a los problemas de las CyMAT impuestas; (d) propiciar la vigilancia del medio ambiente de trabajo y de la salud de los trabajadores; (e) asesorar a los trabajadores acerca de los mecanismos disponibles para la asistencia, rehabilitación y recalificación en caso de accidentes de trabajo y enfermedades profesionales; (f) informar acerca de los derechos, obligaciones y legislación vigente en materia de salud y seguridad en el trabajo; (g) identificar los distintos componentes de la seguridad social y su función en materia de riesgos del trabajo; (h) capacitar a los trabajadores en materia de CyMAT; (i) aplicar los conceptos de la Carrera Municipal de Profesionales de la Salud a la salud laboral; y por último (j) generar técnicas de negociación para la mejora continua de las Condiciones y Medio Ambiente de Trabajo.

De este modo se definió que el perfil del Delegado en CyMAT permitiera, al final del curso, (1) evaluar los riesgos en un marco de comprensión del proceso salud-enfermedad; (2) informar sobre los aspectos del sistema de la Seguridad Social - y sus subsistemas - que determinan la prevención, la atención y la reparación de las Enfermedades Profesionales y los Accidentes de Trabajo; y (3) facilitar las técnicas de negociación sindical para el mejoramiento de las CyMAT.

Los contenidos de enseñanza se dividieron en cinco ejes temáticos que determinaron sendos módulos, a saber:

Módulo I: "El trabajo como determinante del proceso de salud y enfermedad" cuyos principales contenidos fueron: Salud y Trabajo: El trabajo como determinante del proceso salud - enfermedad; Dimensiones epidemiológicas nacionales e internacionales; Condiciones y Medio Ambiente de Trabajo (CyMAT). Enfermedades relacionadas con el trabajo. Enfermedades profesionales. Accidentes de trabajo.

Módulo II: "Riesgos para la salud de los trabajadores de la salud" que incluyó: Riesgo, clasificación y evaluación. Riesgos para la salud de los trabajadores de la Salud: anestésicos, esterilizantes, citostáticos, antisépticos, asbestos, radiaciones ionizantes y no ionizantes, condiciones termohigrométricas, ruido tuberculosis en el Sector Salud, hepatitis virales, Virus de la Inmunodeficiencia Humana, Hantavirus, Chagas, Brucelosis, Citomegalovirus, Riesgos tecnológicos y de seguridad, características constructivas de los lugares de trabajo, instalaciones, riesgo de incendio, seguridad en maquinas y herramientas, riesgo eléctrico, ventilación, iluminación, riesgos psicosociales del trabajo, organización del trabajo, contenido del trabajo, comunicación, estilos de conducción, jornada de trabajo, trabajo por turnos, guardia médica, sistemas de remuneración, lesiones por posiciones forzadas y gestos repetitivos, 
fatiga, carga mental, carga psíquica, estrés laboral, burn-out, violencia en el trabajo.

Módulo III: "Estrategias de intervención en salud laboral", donde se trabajaron conceptos tales como: Vigilancia del Medio ambiente de trabajo, Mapas de riesgos, listas de Cotejo, Concentraciones máximas permitidas, Brigadas de primera intervención, Planes de evacuación, Simulacros, Vigilancia de la salud de los trabajadores, Exámenes de salud, historia clínica ocupacional, Accidentalidad, Investigación de accidentes, Árbol de causas y Encuestas de clima laboral.

Módulo IV: "Instrumentos normativos"; en este módulo se incluyó el abordaje de cuestiones tales como: Servicios de salud en el trabajo. Convenio 161 y recomendación 171 de OIT. Legislación nacional: Ley de Higiene y Seguridad en el trabajo (19587) Ley de Riesgos del Trabajo (24557). Sistema de riesgos del trabajo. Aseguradoras de Riesgos del Trabajo. El rol de la Superintendencia de Riesgos del Trabajo. La accesibilidad de los trabajadores al sistema de riesgos del trabajo .Procedimiento de denuncia. Procedimiento en caso de discrepancia. Comisiones médicas

Por último, el Módulo V: "Herramientas para la negociación sindical", abarcó conceptos acerca de la situación de los trabajadores de la Salud de la Ciudad de Buenos Aires, La Carrera Municipal de Profesionales de la Salud, La negociación gremial en condiciones de trabajo, Bases de datos, Fuentes de consulta.

El curso se desarrolló bajo la modalidad presencial, de carácter teórico práctico, con una duración de 200 horas cátedra distribuidas en 120 teóricas y 80 prácticas entre los meses de Abril y Diciembre de 2007. Las actividades teóricas, consistentes en seminarios y clases teóricas, se desarrollaron en el salón principal de actos de la AMM, con una frecuencia semanal en horario de trabajo matinal, en clases de 4 horas.

Las actividades prácticas se desarrollaron en los hospitales donde trabajan los participantes bajo tutela de la coordinación del curso. Sus objetivos y contenidos estuvieron vinculados a los ejes temáticos de cada módulo.

\section{Producción grupal y evaluación general}

A medida que se desarrollaron los contenidos también se realizaron actividades prácticas. En el primer trabajo práctico los participantes lograron visualizar la interacción entre salud y trabajo en el ámbito laboral cotidiano. En el segundo los futuros delegados en CyMAT relevaron los riesgos presentes en los servicios hospitalarios. Una tercera actividad en terreno posibilitó que los participantes pudieran valorar las condiciones y medio ambiente de trabajo utilizando la metodología del mapa de riesgos. En otras dos actividades prácticas se logró que los futuros delegados de prevención en CyMAT elaboraran una estrategia de intervención gremial destinada a modificar los factores de riesgo existentes en su ámbito laboral y por fin que pudiesen comunicar el impacto de las CyMAT en la salud de los trabajadores.

Estos trabajos prácticos, resueltos en grupos, se discutieron en plenarios con la participación de todos los alumnos y docentes, produciéndose de ese modo puestas en común de conceptos y métodos, lo cual estimuló el trabajo en equipo y la integración gremial.

La AMM dispuso un mecanismo de acceso gratuito a la bibliografía, que en formato digital, fue alojada en un espacio especialmente dedicado al curso en la página Web institucional. Los inscriptos al curso pudieron acceder al mismo, mediante una clave confidencial entregada a cada alumno.

La evaluación del aprendizaje tuvo dos componentes: uno de proceso y otro de resultado. El primero fue evaluado por los docentes a partir de las observaciones de la participación e intervenciones de los alumnos. Para el segundo, se valoró el logro de los objetivos educacionales propuestos para cada módulo a partir de la actividad práctica.

Por otro lado, se llevó a cabo una evaluación del curso por parte de los alumnos a través de una encuesta. La misma constó de una evaluación general del mismo mediante las siguientes preguntas: 1 . En términos generales, ¿el curso le pareció...? Muy interesante, Interesante, Poco interesante, Nada interesante; una evaluación de los contenidos del curso a través de 3 preguntas: 2. ¿Los contenidos del curso fueron...? Muy buenos, Buenos, Regulares, Malos, y dos pregunta abiertas: 2.1 Detalle los temas del curso que le resultaron de mayor utilidad para implementar en su lugar de trabajo y 2.2. Mencione aquellos temas que le parecieron de escasa utilidad; valoración de los docentes, de sus estrategias y de las herramientas operacionales del curso a través de 8 preguntas, 5 de ellas semiestructuradas, 1 dicotómica, y 2 abiertas: 3. ¿Qué valoración hace de las 
estrategias docentes? Muy buenas, Buenas, Regulares, Malas; 4. ¿La dinámica de los trabajos prácticos fue...? Muy buenas, Buenas, Regulares, Malas; 5. En términos de calidad y relevancia ¿el material bibliográfico disponible fue....? Muy bueno, Bueno, Regular, Malo; 5.1.¿Y en términos de volumen? Excesivo, Suficiente, Insuficiente; 6. El sistema de intercambio informático (página web y correo electrónico) ¿Le resultó...? Muy Práctico, Práctico, Poco práctico, Ineficaz; 7. El horario del curso ¿Le resultó conveniente? Si, No, Porqué?; 8. Detalle los aspectos del curso que, a su entender, fueron los más positivos, y 9. Detalle aquellos aspectos del curso que considera negativos.

La información se obtuvo voluntariamente y su tratamiento fue confidencial siguiendo las normas del Acta de Helsinki y sus enmiendas.

\section{RESULTADOS}

No se tienen noticias de que existan experiencias similares a la presentada en el sector de los trabajadores de la salud, particularmente entre profesionales, en la República Argentina.

A través de las observaciones iniciales a cargo del grupo coordinador se destacaron emergentes en el discurso de los participantes que delimitaron un diagnóstico de situación.

De este modo, al comienzo del curso, varios delegados se refirieron al rol gremial en la defensa de la salud laboral y a las dificultades que esta tarea les presentaba: “...habría que asumir la ignorancia que como dirigentes sindicales se tiene [respecto a los temas de CyMAT]..."; o también: "La Universidad no nos formó como trabajadores sino como médicos...".

La mención a las deficientes y dificultosas condiciones de trabajo fueron una constante a lo largo del curso. No obstante, emergieron apreciaciones acerca de los beneficios del trabajo médico en los hospitales porteños respecto al sector privado. En este sentido, la estabilidad del empleo, la existencia de una carrera profesional y la posibilidad de agremiarse fueron algunos de los aspectos citados como ventajosos. Al respecto, un participante dijo: “...qué bueno es estar acá..." [refiriéndose a la posibilidad de contar con este curso de formación sustentado desde el sindicato] y también . “...la Carrera [municipal de profesionales de la salud] tiene previstos muchos aspectos [en relación con el tema de salud y seguridad...] que están pendientes..."
El curso se constituyó así en un ámbito de intercambio y construcción de lazos, a través de un proceso gradual de toma de conciencia y adquisición de conocimientos por parte de los participantes. Dicho proceso se reflejó en sus relatos; si al inicio del curso se sostenía que "...Las enfermedades profesionales eran de otros, no podían sucedernos a nosotros..." y existía la sensación de que "...los médicos tenemos obligaciones y prohibiciones pero carecemos de derechos...", sobre el final expresaban “...Hace más de 20 años [que trabajo] en el Hospital y recién ahora tomé conciencia de las CyMAT..." para acordar que "nuestra salud no vende, se defiende".

De los 65 inscriptos, 58 finalizaron y aprobaron el curso.

La evaluación general del curso que hicieron los alumnos, mediante la encuesta ya citada, arrojó los siguientes resultados: de 52 alumnos respondedores (el 89,6\% del total de inscriptos) el curso fue percibido como "muy interesante" en un $77 \%$ de los casos, e "interesante" para el $23 \%$ de los mismos. No se registraron respuestas correspondientes a las categorías "poco interesante" o "nada interesante".

Los contenidos del curso fueron evaluados positivamente por el mismo universo, concentrándose las respuestas entre las categorías de "muy buenos" ( $72 \%$ de los casos) y "Buenos" (28\% restante).

Las estrategias docentes fueron consideradas principalmente "Muy buenas" (62\%) y "Buenas" $(33 \%)$; mientras que un $5 \%$ de los alumnos las consideraron "Regulares". En consonancia con estos hallazgos, la dinámica de los Trabajos Prácticos fue percibida como "Muy buena" por un $66 \%$ de los participantes, como "Buena" en un 31\% y como "Regular" en $3 \%$.

La bibliografía, estuvo compuesta por 4 Recomendaciones de Agencias Internacionales no OIT, 5 Revisiones, 5 Convenios y Recomendaciones OIT, 7 Trabajos Originales y 9 Normativas Nacionales. La misma fue categorizada como "Muy buena" en el $79 \%$ de los casos y como "Buena" en el $21 \%$ restante. En relación con su volumen ésta fue caracterizada como "Suficiente" por la mayoría de los respondedores (73\%), "Excesiva" por el $24 \%$ e "Insuficiente" sólo por el 3\% restante.

Es interesante el impacto que tuvo el mecanismo de intercambio informático implementado en el curso. El mismo fue evaluado como "Muy práctico" y "Práctico" por el 95\% de los participantes. Dicho 
sistema no sólo incluyó el acceso a la bibliografía, sino que abundó en la comunicación y el intercambio de consultas de los participantes hacia los coordinadores en temas ligados a los contenidos del curso, fechas y programación de actividades; además de fomentar los vínculos interpersonales

Otro ítem estuvo referido al horario de las clases y talleres, el cuál fue categorizado en el $95 \%$ de los casos como conveniente. No se trata de un dato menor, ya que este porcentaje denota el beneficio de considerar aspectos formativos ligados al trabajo dentro del horario de dicho trabajo.

Por último, se han identificado emergentes cualitativos de relevancia: como elementos positivos del curso los participantes han mencionado la toma de conciencia de los riesgos a los que se encuentran expuestos los profesionales médicos, el haber establecido vínculos y adquirido conocimiento de las realidades de los otros hospitales, y finalmente, el reconocimiento de la responsabilidad del empleador. Los aspectos destacados por los asistentes como más negativos fueron el desplazamiento geográfico para los trabajos prácticos (que se realizaron en el Polideportivo de la AMM 5\%) y otro de carácter autocrítico: la dinámica impuesta por los propios concurrentes (impuntualidad, uso de teléfonos celulares 3\%). Entre las sugerencias figuraron el dictado de una segunda instancia del curso y su extensión a otros compañeros, junto al pedido a las autoridades de la instauración de Servicios de Salud y Seguridad en el Trabajo y de la conformación de comités de CyMAT en cada centro hospitalario.

\section{DISCUSIÓN}

El binomio salud - trabajo se articula a lo largo de la historia de la humanidad y particularmente desde los cambios en la producción derivados de la Revolución Industrial, que introduce modificaciones en las exposiciones de los trabajadores, como nunca antes se había imaginado.

Esta articulación ha evolucionado a tal punto que fue considerado como el inicio del establecimiento de las bases teóricas para la ideación y desarrollo de los incipientes sistemas de seguridad social en la Europa de mediados del siglo XIX.

Plasmada esta relación a lo largo de toda la Modernidad y de la Era Contemporánea, la salud y el trabajo llegaron a ser reconocidos como derechos inalienables de las personas, hasta un punto en que la pérdida de uno de ellos en ejercicio del otro resul- ta inaceptable.

En el Sector Salud, y particularmente en el caso de los profesionales médicos, se vienen observando enfermedades y discapacidades derivadas de la exposición a un importante número de agentes de riesgo, pese al limitado desarrollo de los sistemas de registro y notificación. La complejidad de las acciones y relaciones de estos agentes determinan un perfil de difícil intervención. En efecto, el desconocimiento o la naturalización de los riesgos del trabajo, la falta de conformación de los comités mixtos de CyMAT, y la histórica escasa intervención gremial paritaria para el mejoramiento de dichas condiciones, perpetúan - cuando no incrementan - la vulnerabilidad de los trabajadores médicos.

De acuerdo con la bibliografía actual es posible pensar una reconversión conceptual de los factores de exposición, desde una mirada clásica funcionalista, a otra que considera a estos factores como una imposición, desde la Epidemiología Social. Es aquí donde adquiere mayor relevancia la conceptualización de Trabajo Decente, en la que se afirma que éste “...resume las aspiraciones de los individuos en lo que concierne a sus vidas laborales, e implica oportunidades de obtener un trabajo productivo con una remuneración justa, seguridad en el lugar de trabajo y protección social para las familias, mejores perspectivas para el desarrollo personal y la integración social, libertad para que los individuos manifiesten sus preocupaciones, se organicen y participen en la toma de aquellas decisiones que afectan a sus vidas, asi como la igualdad de oportunidades y de trato para mujeres y hombres... vii"..

En este sentido, la respuesta de la organización gremial a una demanda establecida organizadamente, y resultante de un encuentro institucional, surge como una acción concreta, equitativa, colectiva y sostenible en el tiempo.

La complejidad de las exposiciones de los trabajadores del Sector Salud - o imposiciones - , en particular las de los médicos, es notable. Existen diversos tipos de agentes a los que se dan estas exposiciones: contaminantes químicos (como Oxido de Etileno, Citostáticos, Gases Anestésicos, Formol, Glutaraldehído, Hipoclorito de sodio, Yodo, Ac. Acético, Mercurio, Xileno, Tolueno, Freones, Metacrilato, y otros menos frecuentes etc.), físicos (como Ruidos y vibraciones, Carga térmica, Radiación Ionizante o no Ionizante, Ventilación, Iluminación, gases comprimidos), o los clásicamente relaciona- 
dos agentes biológicos (Hepatitis, Tuberculosis, VIH, Citomegalovirus, Herpes simple, Tétanos, Rubéola, Sarampión, Hantavirus, Brucella, Cándida, etc.). A estos también hay que sumar los riesgos de incendio, eléctrico, maquinarias y herramientas, pisos, escaleras, aberturas, iluminación, ventilación, y aquellos derivados de la carga física y mental de trabajo con su secuela de fatiga y estrés y los llamados riesgos psicosociales (tiempo de trabajo, ritmos de trabajo, comunicación, estilos de conducción o gestión de la fuerza de trabajo, forma de remuneración, y la violencia, en cualquiera de sus formas).

El curso de formación de delegados en CyMAT expone en sus contenidos dicha complejidad y ha abundado en una temática de orden técnico hasta alcanzar niveles de percepción satisfactoria según lo revelan los resultados de la evaluación presentada. Sin embargo, conjuntamente con las cuestiones consideradas clásicamente técnicas, se ha producido una construcción del aprendizaje al mismo tiempo que se desarrolló una praxis político-gremial que ha posibilitado lograr emergentes tales como el mantenimiento de la formación, su apertura a más compañeros y la creación de ámbitos instrumentales para el mejoramiento de las condiciones de trabajo. En este sentido, la conformación de una Comisión Consultiva

\section{BIBLIOGRAFIA}

i. Ramazzini B. De morbis artificum diatriba. $1^{\text {a }}$ Edición. Buenos Aires: Sociedad de Medicina del trabajo de la Provincia de Buenos Aires, 1989.

ii. Darham GHAI. Trabajo decente: concepto e indicadores. Revista Internacional del Trabajo [on line] 2003; 122 (2): 125-59. [citado el 25 de mayo de 2008]. Disponible en la World Wide Web:

http://portal.oit.or.cr/index.php?option $=$ com_staticxt\&staticfile $=$ genero/trabajo $\% 20$ decente $\% 20$ indicadores.pdf

iii. Clerc J.M. (editor). Introducción a las condiciones y el medio ambiente de trabajo. Geneve: OIT, 1987.

iv. 1ra Jornada "El rol de las Entidades Gremiales en la Protección de la Salud y Seguridad de los Trabajadores" 19 de Octubre de 2006. permanente de CyMAT en el seno del Comité Ejecutivo de la AMM y el reclamo a las autoridades por la conformación de servicios de Salud y Seguridad y comités paritarios de CyMAT en cada hospital, representan dos acciones inmediatas emanadas del propio proceso de enseñanza-aprendizaje.

Es destacable asimismo el valor de las acciones interdisciplinarias llevadas a cabo por el grupo coordinador del curso de formación de delegados de prevención en CyMAT. En efecto, los objetivos, las acciones y los resultados dan cuenta de la convergencia de matrices disciplinares, superando una mirada ligada a la multidisciplina, hacia un objeto que se constituye así como nuevo, distinto de la obtención de cuotas de saber a cargo de cada disciplina por separado ${ }^{\text {viii. }}$.

Por último esta experiencia nos permite verificar que es posible generar conocimiento traducido en acciones gremiales concretas respecto a los riesgos del lugar de trabajo mediante el compromiso de los principales sujetos: los trabajadores médicos

En definitiva, se trata de ganarse la vida trabajando, no de perderla en el intento....

v. OIT. C155-Convenio sobre seguridad y salud de los trabajadores, 1981 [on line] [citado el 25 de mayo de 2008]. Disponible en la World Wide Web en: http://www.ilo.org/ilolex/cgi-lex/convds.pl

vi. OIT. R164 Recomendación sobre seguridad y salud de los trabajadores, 1981[on line] [citado el 25 de mayo de 2008]. Disponible en la World Wide Web en: http://www.ilo.org/ilolex/cgi-lex/convds.pl

vii. OIT; [on line] [citado el 25 de mayo de 2008]. en: http://www.ilo.org/public/spanish/decent. htm

viii. Pedro Luis Sotolongo Codina y Carlos Jesús Delgado Díaz. La revolución contemporánea del saber y la complejidad social : hacia unas ciencias sociales de nuevo tipo. 1a edición. Buenos Aires : Consejo Latinoamericano de Ciencias Sociales CLACSO, 2006. 